\title{
Colocación de un tornillo transfisario medial en la cadera espástica de niños con parálisis cerebral. Eficacia y seguridad
}

\author{
Francisco Praglia, "\# Florencia Lucioni, ${ }^{*}$ Juan Pablo Cucchiara \\ "Servicio de Cirugía Ortopédica Pediátrica, Departamento Materno Infantil, Hospital Militar Central "Cir. Mayor Dr. Cosme Argerich", \\ Ciudad Autónoma de Buenos Aires, Argentina \\ "Unidad de Ortopedia Infantil, Hospital Nacional "Prof. Alejandro Posadas", Buenos Aires, Argentina
}

\begin{abstract}
RESUMEN
Introducción: En estudios experimentales y clínicos, los resultados con el tornillo excéntrico transfisario medial para producir varo fueron alentadores. El propósito de este estudio fue determinar si colocar un tornillo en el fémur proximal es un gesto eficaz y seguro en caderas espásticas de niños con parálisis cerebral. Materiales y Métodos: Se incluyó a pacientes con parálisis cerebral infantil y caderas en riesgo. Se compararon la serie A: pacientes con liberación de partes blandas más colocación de un tornillo excéntrico transfisario medial en el fémur proximal y la serie B: pacientes solo con liberación de partes blandas. Se determinaron el test de Rang, el índice de migración de Reimer, el ángulo cervicodiafisario y las complicaciones, antes de la cirugía y después. Resultados: Se operó a 18 pacientes (36 caderas): 10 de la serie A y 8 de la serie B, con una mediana de edad de 51 meses y una mediana de seguimiento, de 3 años. Al comparar por delta de medianas todas las variables preoperatorias y posoperatorias, hubo una diferencia estadísticamente significativa solo en el delta de mediana del ángulo cervicodiafisario de las caderas izquierdas (-5 vs. 0, p 0,02). Conclusiones: La liberación de partes blandas es eficaz para prevenir la luxación de la cadera espástica. La colocación de un tornillo transfisario excéntrico en la cadera espástica no produjo cambios y no fue inocua. La hemifisiodesis medial de la cadera es una atractiva solución teórica para tratar los problemas ocasionados por el valgo excesivo, pero se requieren más estudios.
\end{abstract}

Palabras clave: Cadera espástica; tornillo; fémur proximal; niños.

Nivel de Evidencia: III

Medial transphyseal screw placement for spastic hip treatment in children with cerebral palsy. Effectiveness and safety

\section{ABSTRACT}

Introduction: Animal and clinical studies have shown promising results for the varus-producing placement of a medial eccentric transphyseal screw. The purpose of this study was to establish if the placement of a screw in the proximal femur is an effective and safe approach for spastic hips in children with cerebral palsy (CP). Materials and Methods: We compared two series of pediatric CP patients (Gross Motor Function Classification System [GMFCS] III, IV and V) with "hips at risk." Series A patients were treated with soft-tissue release plus a medial eccentric transphyseal screw in the proximal femur. Series B patients were only treated with soft-tissue release. Patients were evaluated pre- and postoperatively to determine their Rang test score, Reimer's migration index (MI), diaphyseal cervical angle, and complications. Results: From a total of 18 patients operated, 36 hips, $55 \%$ (10) belonged to the A Series and $45 \%(8)$ to the B Series. The median age was 51 months (IQR, 41-108). The median follow-up was 3 years (IQR, 2.4-5.8). The comparative analysis of all preoperative and postoperative variables yield only one statistically significant difference: the median left hip diaphyseal cervical angle ( -5 vs. $0, P$ 0.02). Conclusions: The release of soft tissues was effective to prevent the spastic hip dislocation. The placement of medial eccentric transphyseal screw in spastic hips produced some complications and no beneficial changes. Medial hemiphysiodesis of the hip remains nothing but an attractive theoretical solution for the treatment of problems caused by excessive valgus. However, further studies are warranted.

Key words: Spastic hip; screw; proximal femur; children.

Level of Evidence: III 


\section{INTRODUCCIÓN}

La parálisis cerebral infantil (PCI) es una alteración neurológica secundaria a una lesión en un cerebro inmaduro, que ocurre antes de los 5 años de vida o hasta cinco años después del nacimiento. ${ }^{1}$ En estos pacientes, el cuadro primario no es solo la hipertonía, ya que existen otras afecciones primarias, como pobre equilibrio, debilidad y pérdida del control selectivo. El tipo más frecuente de PCI es el espástico. El Sistema de Clasificación de la Función Motora Gruesa (Gross Motor Function Classification System, GMFCS) es el método más utilizado en la actualidad para clasificar a los pacientes con PCI, basándose en las capacidades funcionales y las limitaciones. ${ }^{2}$ Es el mejor método para comunicar la gravedad y el pronóstico de la deambulación. La fisiopatología de la displasia de la cadera espástica es distinta de la de la displasia del desarrollo. Las caderas espásticas son el resultado del desequilibrio muscular y las deformidades óseas secundarias agravadas por la falta de carga. La espasticidad de los aductores y flexores, con debilidad relativa de extensores y abductores, provoca mayor anteversión y valgo del cuello femoral con aumento progresivo de la displasia acetabular (habitualmente anterolateral). La oblicuidad pelviana secundaria o primaria influye también en la producción de la inestabilidad articular..$^{3-5}$ La evolución natural de la displasia espástica de la cadera es la progresión gradual durante varios años. Cuanto más severa es la PCI, más rápida es la progresión hacia la luxación. ${ }^{5}$ La vigilancia precoz y el tratamiento preventivo de la luxación en pacientes con caderas espásticas buscan evitar y retrasar las cirugías óseas. La cadera luxada en pacientes con PCI menoscaba la calidad de vida, gatilla un círculo vicioso de dolor, irritabilidad y aumento de la espasticidad. Como consecuencia de la luxación de caderas y de las deformidades de la columna, el cuidador puede tener enormes dificultades para sentar al niño, realizarle la higiene perineal y cambiarle la ropa. Así mismo, trasladar a un niño con contracturas articulares de una silla de ruedas a la cama puede ser una tarea que requiera gran demanda física por parte del cuidador. Las escaras por decúbito condicionan un mayor tiempo en decúbito dorsal, que ocasiona más reflujo gastroesofágico y broncoaspiración. ${ }^{3,4}$ Los tiempos óseos en cirugías ortopédicas tanto reconstructivas como paliativas no están exentos de complicaciones y agregan morbilidad a estos pacientes. ${ }^{4,5}$ La liberación de partes blandas (LPB) precoz en pacientes con caderas en riesgo es muy eficaz; sin embargo, es frecuente que la subluxación recurra durante el crecimiento del niño. ${ }^{4} \mathrm{El}$ uso del crecimiento guiado en pacientes esqueléticamente inmaduros es conocido desde hace varios años. Los desejes en rodilla, tobillo y trocánter mayor suelen lograr buenos resultados. ${ }^{6-11}$ La hipótesis de modular el crecimiento medial del fémur proximal resulta muy atractiva, se podrían corregir problemas frecuentes de la cadera infantil secundarios al valgo excesivo, con un gesto mínimamente invasivo. En estudios con animales, la colocación de un tornillo excéntrico transfisario augura resultados alentadores en la producción de varo. ${ }^{12,13}$ Recientes ensayos clínicos también obtuvieron buenos resultados. ${ }^{14-17}$

El propósito de este estudio fue determinar si la colocación de un tornillo de 4,5 $\mathrm{mm}$ de diámetro, excéntrico medial transfisario en el fémur proximal de pacientes con PCI es eficaz para producir cambios morfológicos en sus caderas.

\section{OBJETIVOS GENERALES}

- Determinar si la colocación de un tornillo medial en la cadera es un gesto coadyuvante útil a la LPB para mejorar la displasia de la cadera espástica a corto plazo.

- Determinar si la colocación de un tornillo medial en la cadera es un gesto seguro en pacientes con PCI.

\section{MATERIALES Y MÉTODOS}

Se diseñó un estudio retrospectivo que comparó dos series de casos. Se incluyó a niños con parálisis cerebral GMFCS III, IV y V, con una o ambas caderas en riesgo, tratados entre 2009 y 2014, en tres instituciones diferentes, por el mismo equipo quirúrgico. Se consideró "cadera en riesgo" si el paciente tenía parálisis cerebral y menos de 7 años, un test de abducción de Rang $<30^{\circ}$ y una radiografía de caderas de frente con un índice de migración de Reimers (IR) $>25 \% .{ }^{4}$ Todos fueron sometidos a un procedimiento quirúrgico preventivo de la cadera espástica en riesgo. El protocolo quirúrgico consistió en una tenotomía del aductor mediano de caderas y una tenotomía del recto interno. $\mathrm{Si}$, luego de estos gestos, el test de Rang era $<45^{\circ}$, se procedía a una tenotomía del aductor menor y una neurectomía de la rama anterior del nervio obturador más tenotomía del tendón del psoas en niños no deambuladores, pero, en deambuladores, se efectuó el deslizamiento miofacial de dicho tendón. ${ }^{4}$ Luego se inmovilizaban ambos miembros inferiores por 15 días con yeso tipo Ducroquet (Figura 1). A este procedimiento lo llamamos LPB.

Se analizaron dos series: la serie A incluyó a pacientes sometidos a LPB y colocación de un tornillo de 4,5 mm de diámetro, canulado, excéntrico transfisario medial en el fémur proximal, de forma percutánea, guiado con intensificador de imágenes, en el mismo acto quirúrgico. 


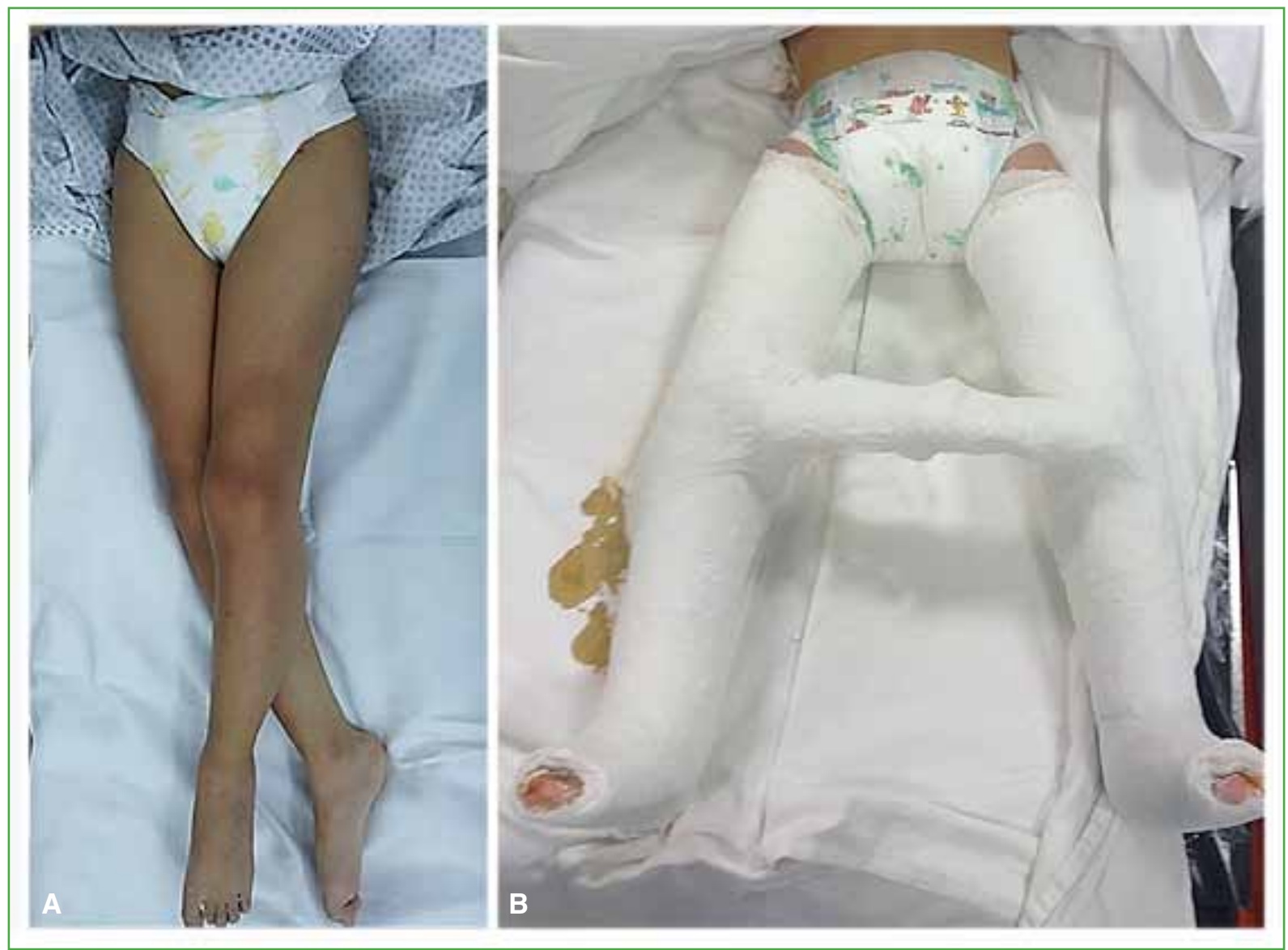

Figura 1. Paciente de 6 años con parálisis cerebral infantil GMFCS IV. A. Imagen preoperatoria. B. Posoperatorio inmediato con yeso inguinopedio bilateral que abduce ambas caderas.

La cirugía se practicó con el paciente en posición supina, bajo anestesia general, a través de una incisión de aproximadamente $2 \mathrm{~cm}$ en el lado lateral del muslo, distal a la fisis del trocánter mayor. Se introdujo un alambre guía de Kirschner a través del cuello femoral pasando la fisis. El alambre guía se colocó en el cuadrante inferomedial (la mitad medial de la cabeza femoral en la vista anteroposterior) con asistencia de un intensificador de imágenes. Después de perforar el hueso, se insertó un tornillo canulado de $4,5 \mathrm{~mm}$, parcialmente roscado de titanio. Se progresaron tres o cuatro espiras de la rosca más allá de la fisis. Su posición se confirmó por fluoroscopia (Figura 2A). En algunos casos, se realizó una artrografía de la cadera para descartar una migración intrarticular del tornillo (Figura 2B).

La serie B estaba integrada por pacientes sometidos solo a LPB.

\section{Variables de resultados clínicos y radiográficos}

Las variables estudiadas fueron: test de abducción de Rang, dolor, dificultad para el aseo perineal y conformidad de los padres mediante la "Escala del Cuidador" del Instituto de Ortopedia Infantil Roosevelt, de Bogotá, Colombia ${ }^{18}$ que contempla los siguientes dominios: 1) cuidado personal, 2) posicionamiento y transferencias, y 3) confort. Se consideró si hubo otros gestos quirúrgicos concomitantes en un nivel diferente. En las radiografías anteroposteriores de caderas, se midieron el ángulo cervicodiafisario (ACD) y el IR. 


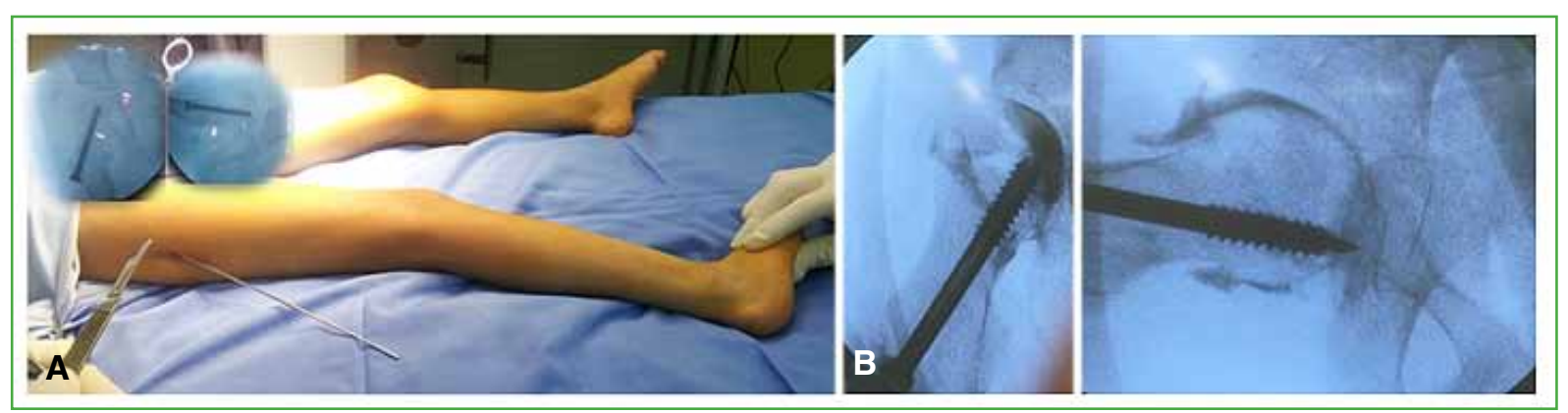

Figura 2. A. El primer paso de la cirugía de crecimiento guiado es corregir la anteversión del fémur con rotación interna de la cadera hasta que se observa el cuello femoral bien desplegado por fluoroscopia. Se coloca el alambre guía. Después de introducir la mecha canulada, se procede a colocar un tornillo canulado de 4,5 mm de diámetro. B. Artrografía de la cadera para descartar una migración intrarticular del tornillo.

\section{Variables de seguridad}

Las complicaciones se cuantificaron según la clasificación de Dindo-Clavien. ${ }^{19}$

\section{Criterios de exclusión}

Los criterios de exclusión fueron: seguimiento $<2$ años, cirugía previa de cadera, antecedente de infección en la cadera y otro cuadro neuromuscular de base diferente de la PCI. Los resultados se analizaron con los estándares de luxación para la cadera espástica, propuestos por Presedo y cols. ${ }^{20}$ IR $<24 \%$, bueno; IR 25-39\%, regular; IR $40-59 \%$, malo e IR $>60 \%$, fracaso.

\section{Análisis estadísticos}

Las variables continuas se expresan como media y desviación estándar; y las categóricas, como porcentaje con su frecuencia absoluta. Para cada serie A (LPB más tornillo medial en el fémur proximal) y B (solo LPB), se compararon los delta de medianas (preoperatorios y posoperatorios), el test de Rang de la cadera derecha y la cadera izquierda, el IR de la cadera derecha y la cadera izquierda, el ACD de la cadera derecha y la cadera izquierda. Para las variables continuas, se utilizó el test de Mann-Whitney y, para las variables categóricas, la prueba de Fisher. Se consideró estadísticamente significativo un valor $\mathrm{p}<0,05$.

\section{RESULTADOS}

La serie A estaba integrada por 10 pacientes (20 caderas) y la serie B, por ocho (16 caderas). En la serie A, había cuatro pacientes GMFCS IV y seis GMFCS V y, en la serie B, dos pacientes GMFCS III, tres GMFCS IV y tres GMFCS V. Ambas incluían 13 niños y 5 niñas. La mediana de la edad era de 51 meses (RIC 41-108). El 39\% (n = 7) fue sometido a un único gesto quirúrgico durante la operación. En el resto, además del tratamiento preventivo para la cadera espástica, se realizaron otros gestos ortopédicos en diferentes niveles, por ejemplo, quimiodesnervación reversible selectiva con onabotulinuntoxin A en isquiotibiales y gemelos, tenoplastias de isquiotibiales o gemelos. El seguimiento promedio fue de tres años (RIC 2.4-4.8) en la serie A y de tres años (RIC 2.4-5.8) en la serie B (Tabla 1). 
Tabla 1. Características de los pacientes

\begin{tabular}{|c|c|c|c|}
\hline & $\begin{array}{c}\text { Total } \\
\mathrm{n}=18\end{array}$ & $\begin{array}{c}\text { Grupo A } \\
\mathbf{n}=\mathbf{1 0}\end{array}$ & $\begin{array}{c}\text { Grupo B } \\
n=8\end{array}$ \\
\hline Sexo masculino & 13 & 8 & 5 \\
\hline Mediana de la edad, meses & 51 (RIC 41-108) & 52 (RIC 43-103) & 57 (RIC 40-108) \\
\hline Cirugía única & $39 \%(7)$ & $40 \%(4)$ & $38 \%(3)$ \\
\hline Complicaciones & $16,6 \%(3)$ & $66,6 \%(2)$ & $33,3 \%(1)$ \\
\hline Seguimiento, años & 3 (RIC 2.4-5.8) & 3 (RIC 2.4-4.8) & 3 (RIC 2.4-5.8) \\
\hline \multicolumn{4}{|l|}{ GMFCS } \\
\hline III & $11 \%(2)$ & 0 & $25 \%(2)$ \\
\hline IV & $39 \%(7)$ & $40 \%(4)$ & $37,5 \%(3)$ \\
\hline $\mathrm{V}$ & $50 \%(9)$ & $60 \%(6)$ & $37,5 \%(3)$ \\
\hline
\end{tabular}

\section{Resultados clínicos}

La mediana del test de Rang de la cadera derecha preoperatoria fue de $28^{\circ}$ (RIC 20-45) en la serie A y $25^{\circ}$ (RIC 23-35) en la serie B. La mediana del test de Rang de la cadera derecha posoperatoria, con seguimiento máximo, fue de $50^{\circ}$ (RIC 45-55) y $52^{\circ}$ (RIC 50-60), respectivamente. El delta de mediana del test de Rang de la cadera derecha fue de $23^{\circ}$ (RIC 15-30) en la serie A y $28^{\circ}$ (RIC 18-35) en la serie B (p 0,3, no significativo).

La mediana del test de Rang de la cadera izquierda preoperatoria fue de $23^{\circ}$ (RIC 20-30) en la serie A y $25^{\circ}$ (RIC 20-45) en la serie B. La mediana del test de Rang de la cadera izquierda posoperatoria, con seguimiento máximo, fue de $45^{\circ}$ (RIC 45-50) y $45^{\circ}$ (RIC 43-55), respectivamente (Tabla 2).

Tabla 2. Mediciones preoperatorias y posoperatorias

\begin{tabular}{|c|c|c|c|}
\hline & $\begin{array}{c}\text { Total } \\
\mathrm{n}=18\end{array}$ & $\begin{array}{c}\text { Grupo A } \\
\mathbf{n}=\mathbf{1 0}\end{array}$ & $\begin{array}{c}\text { Grupo B } \\
\mathbf{n}=\mathbf{8}\end{array}$ \\
\hline Mediana del test de Rang CD preoperatoria & 25 (RIC 20-45) & 28 (RIC 20-45) & 25 (RIC 23-25) \\
\hline Mediana del test de Rang CI preoperatoria & 20 (RIC 20-45) & 23 ((RIC 20-30) & 25 (RIC 20-45) \\
\hline Mediana del IR CD preoperatoria & 27 (RIC 15-74) & 28 (RIC 14-74) & 27 (RIC 23-45) \\
\hline Mediana del IR CI preoperatoria & 30 (RIC 25-83) & 31 (RIC 25-83) & 28 (RIC 23-46) \\
\hline Mediana del ACD CD preoperatoria & 159 (RIC 155-170) & 156 (RIC 155-170) & 160 (RIC 155-165) \\
\hline Mediana del ACD CI preoperatoria & 160 (RIC 150-170) & 162 (RIC 160-170) & 160 (RIC 158-168) \\
\hline Mediana del test de Rang CD posoperatoria & 50 (RIC 50-60) & 50 (RIC 45-55) & 52 (RIC 50-60) \\
\hline Mediana del test de Rang CI posoperatoria & 45 (RIC 45-55) & 45 (RIC 45-50) & 45 (RIC 43-55) \\
\hline Mediana del IR CD posoperatoria & 24 (RIC 18-51) & 24 (RIC 18-51) & 23 (RIC 19-35) \\
\hline Mediana del IR CI posoperatoria & 20 (RIC 18-83) & 20 (RIC 17-41) & 23 (RIC 19-83) \\
\hline Mediana del ACD CD posoperatoria & 156 (RIC 148-170) & 156 (RIC 148-170) & 158 (RIC 155-165) \\
\hline Mediana del ACD CI posoperatoria & 159 (RIC 153-170) & 153 (RIC 150-170) & 160 (RIC 156-170) \\
\hline
\end{tabular}

$\mathrm{CD}=$ cadera derecha, $\mathrm{CI}$ = cadera izquierda, $\mathrm{IR}$ = índice de Reimer, $\mathrm{ACD}$ = ángulo cervicodiafisario. 
El delta de mediana del test de Rang de la cadera izquierda fue de $25^{\circ}$ (RIC 25-25) en la serie A y $20^{\circ}$ (RIC 15-30) en la serie B (p 0,3, no significativo). Los puntajes en la "Escala del Cuidador" 18 disminuyeron (mejoraron) en los tres dominios. Un paciente de la serie A refirió dolor persistente en la cadera izquierda después de la cirugía, por lo que fue necesario extraer los tornillos a los ocho meses de colocarlos. Aun así no hubo diferencias estadísticamente significativas entre las series ( $\mathrm{p} 0,2)$.

\section{Resultados radiográficos}

Cadera derecha

IR. Serie A: mediana preoperatoria $28 \%$ (RIC 14-74), mediana posoperatoria $24 \%$ (RIC 18-51). Serie B: mediana preoperatoria 27\% (RIC 23-45), mediana posoperatoria 23\% (RIC 19-35). El delta de mediana del IR fue 3 (RIC de -12 a 21) en la serie A y -2 (RIC de -9 a 8) en la serie B (p 0,56, no significativo).

ACD. Serie A: mediana preoperatoria $156^{\circ}$ (RIC 155-170), mediana posoperatoria $156^{\circ}$ (RIC 148-170). Serie B: mediana preoperatoria $160^{\circ}$ (RIC 155-165), mediana posoperatoria $158^{\circ}$ (RIC 155-165). El delta de mediana del ACD fue -1 (RIC de -7 a 5) en la serie A y 0 (RIC 0-0) en la serie B (p 0,2, no significativo) (Tabla 2).

\section{Cadera izquierda}

IR. Serie A: mediana preoperatoria 31\% (RIC 25-83), mediana posoperatoria $20 \%$ (RIC 17-41). Serie B: mediana preoperatoria $28 \%$ (RIC 23-46), mediana posoperatoria $23 \%$ (RIC 19-83). El delta de mediana del IR fue de -9 (RIC de -23 a 5) en la serie A y -5 (RIC de -6 a 38) en la serie B (p 0,3, no significativo).

ACD. Serie A: mediana preoperatoria $162^{\circ}$ (RIC 160-170), mediana posoperatoria $153^{\circ}$ (RIC 150-170). Serie B: mediana preoperatoria $160^{\circ}$ (RIC 158-168), mediana posoperatoria $160^{\circ}$ (RIC 156-170). El delta de mediana del ACD fue de -5 (RIC de -10 a 0) en la serie A y 0 (RIC de -2 a 3) en la serie B (p 0,02, diferencia estadísticamente significativa). En todos los casos de la serie A, se observó la migración distal de los tornillos con el crecimiento (Figuras 3 y 4 ).

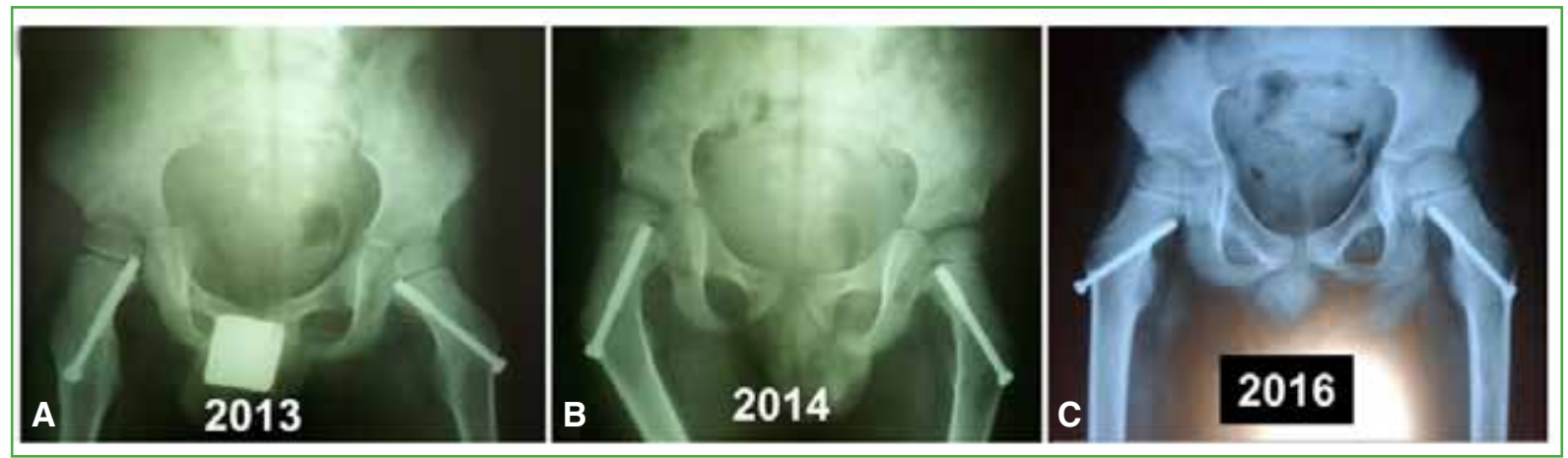

Figura 3. Radiografías de cadera de frente. Paciente de 4 años con parálisis cerebral GMFCS IV. A. Ocho meses después de la cirugía. Se observa una incipiente progresión distal de los tornillos en ambas caderas. B y C. Conforme pasan los años, la progresión es mayor.

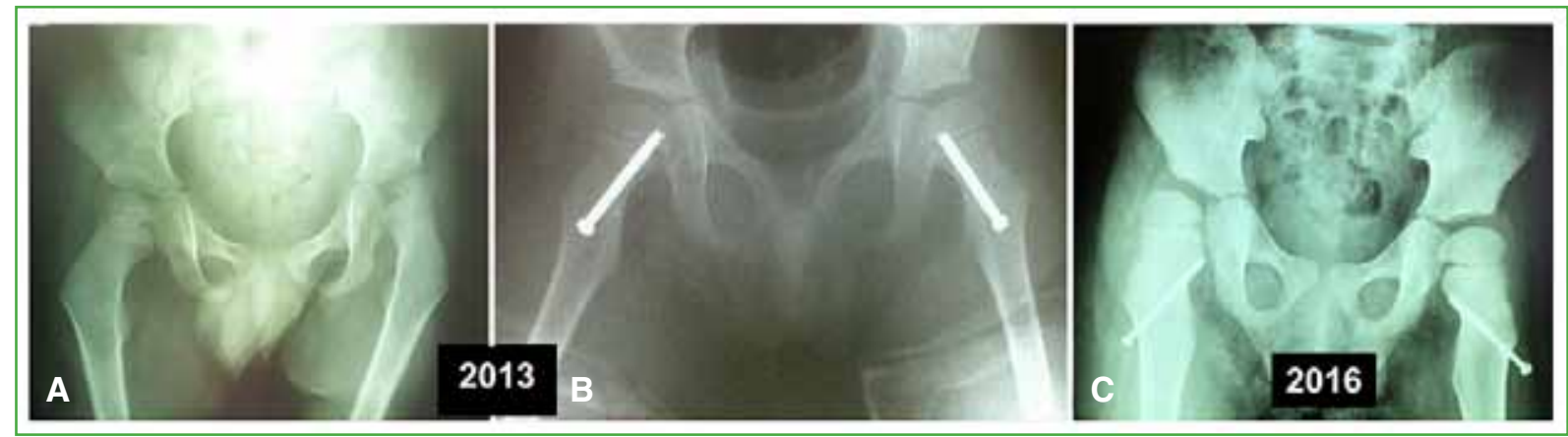

Figura 4. Radiografías de cadera de frente. Paciente con parálisis cerebral GMFCS IV. A. Cuatro años antes de la cirugía.

B. Posoperatorio inmediato. C. Progresión distal de los tornillos en ambas caderas, con el paso del tiempo. 
Considerando el IR y su efecto en la prevención de cirugías óseas, los resultados fueron: serie A: $55 \%$ buenos, $25 \%$ regulares, $15 \%$ pobres y $5 \%$ fracaso; serie B: $50 \%$ buenos, $31 \%$ regulares, $13 \%$ pobres y $6 \%$ fracaso (p 0,1 , diferencia no significativa) (Figuras 5 y 6 ).

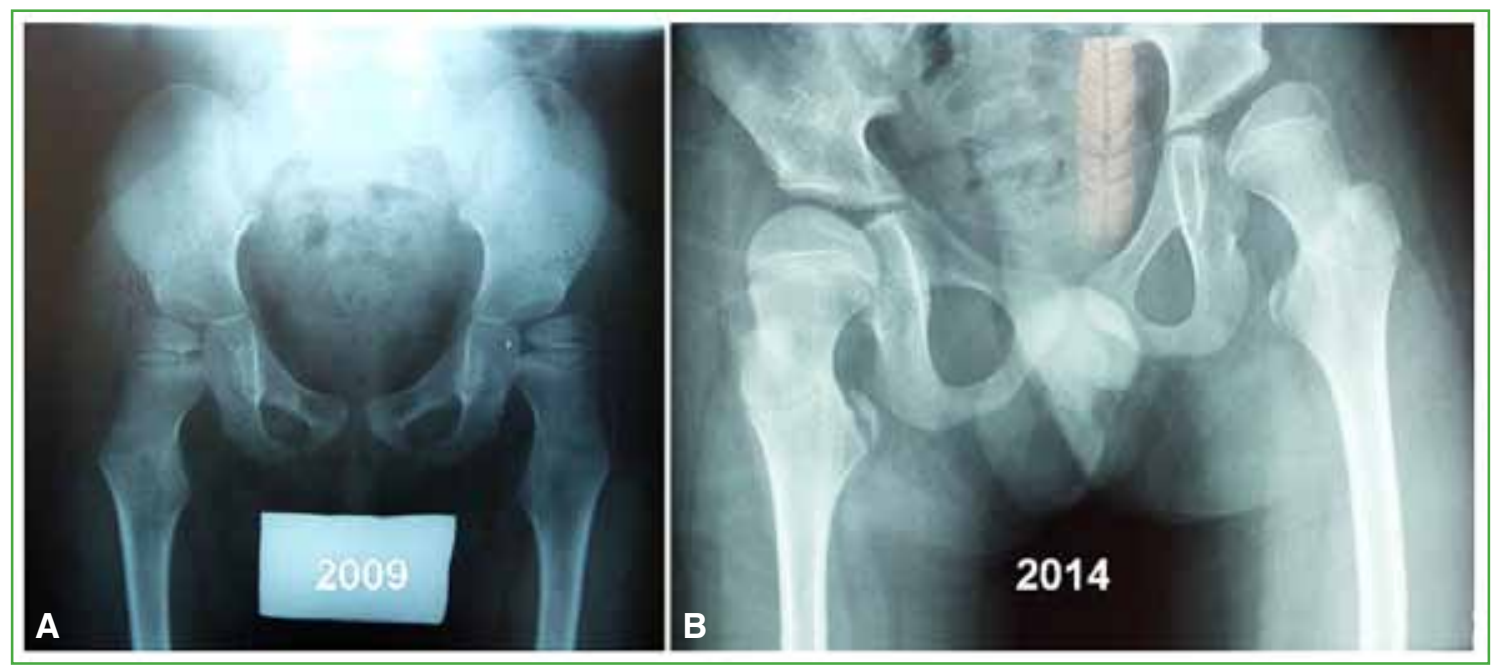

Figura 5. Radiografías de cadera de frente. Paciente de 5 años con parálisis cerebral GMFCS III. A. Ambas caderas en riesgo. B. Cinco años después de la liberación bilateral de partes blandas, las caderas no se luxaron y tienen un índice de Reimer y un ángulo cervicodiafisario estables.

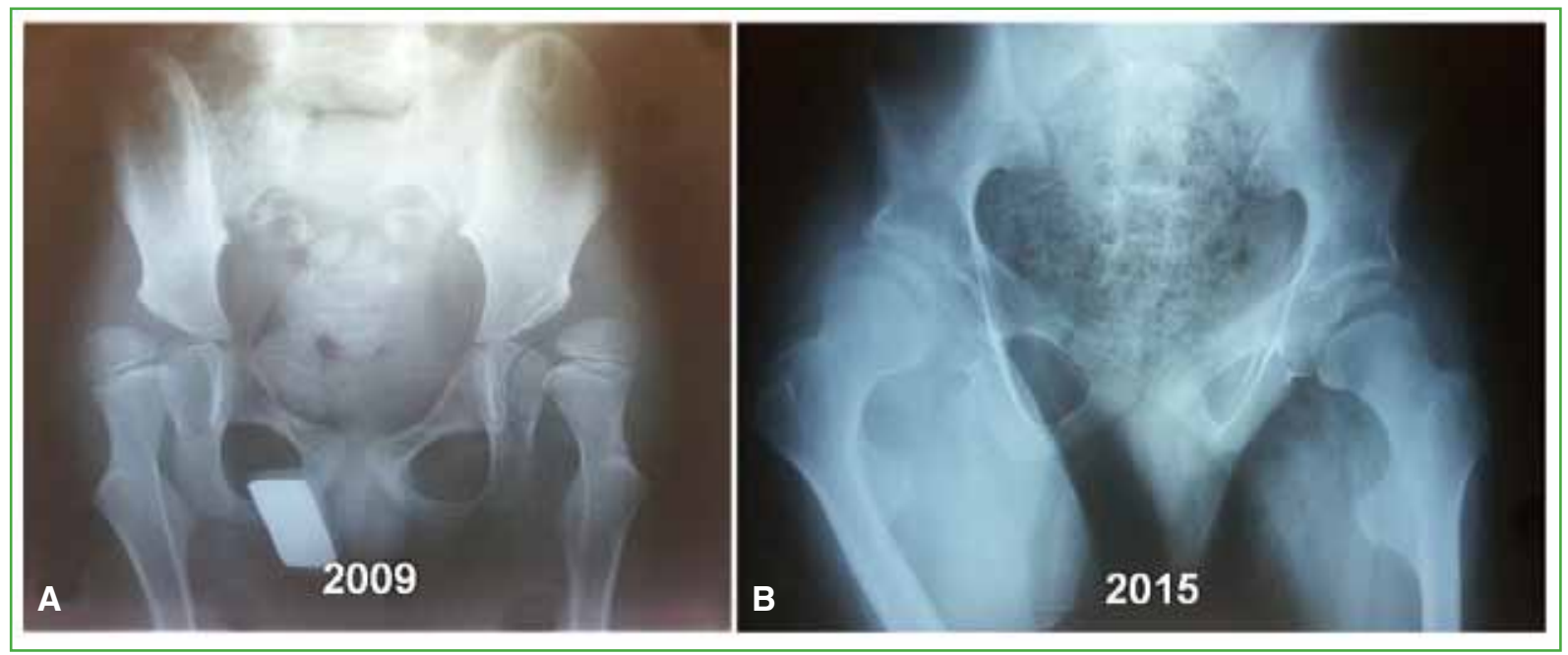

Figura 6. Radiografías de cadera de frente. Paciente de 6 años con parálisis cerebral GMFCS V. A. Ambas caderas con un índice de Reimer $>60 \%$. B. A los 12 años de edad, 5 años después de la liberación bilateral de partes blandas, no solo no se luxaron las caderas, sino que también mejoraron sus índices radiográficos.

\section{Variables de seguridad}

Las complicaciones se cuantificaron con la clasificación de Dindo-Clavien. ${ }^{19}$ En la serie A, hubo dos complicaciones: una clasificada como tipo III $\mathrm{b}$, dolor en la cadera izquierda que requirió el retiro del tornillo transfisario a los ocho meses de la cirugía (Figura 7), y otra complicación clasificada como I, una ruptura de la cabeza del tornillo durante la colocación y fue necesario colocar otro paralelo. En la serie B, hubo una infección superficial de la herida quirúrgica, clasificada como tipo II. La diferencia entre las series no fue estadísticamente significativa (p 1). 


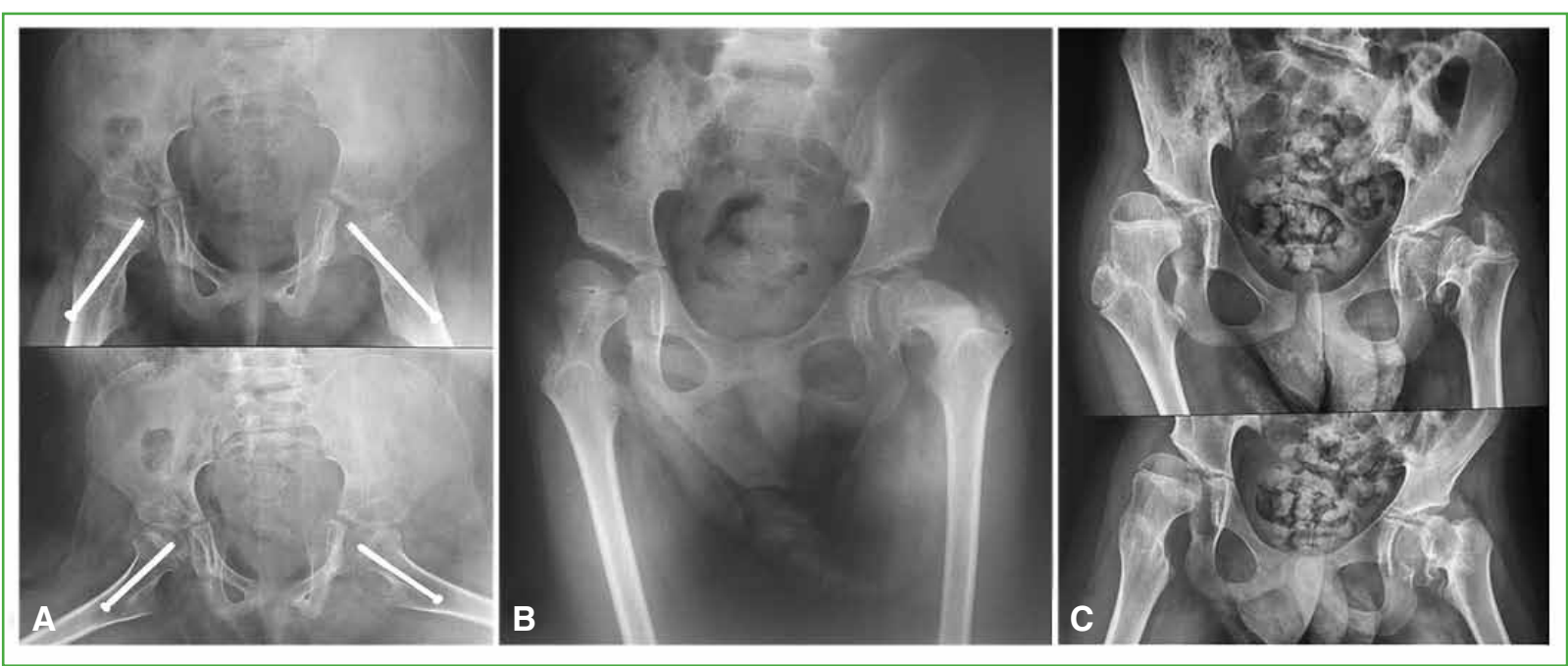

Figura 7. Paciente con parálisis cerebral, cuadriparesia espástica, GMFCS IV. A. Radiografías de cadera de frente y de perfil, posoperatorio inmediato. B. Radiografía de cadera de frente tomada a los 20 meses de retirar los tornillos de ambas caderas. Se observa el cierre fisario medial definitivo de la cadera izquierda, con deformidad en varo. C. Radiografías de cadera de frente y de perfil a los 4.5 años de la cirugía. Cadera izquierda: coxa breva y vara con ascenso del trocánter mayor. Cadera derecha: valga anteversa con progresión de la displasia espástica. Índice de Reimer $>50 \%$.

\section{DISCUSIÓN}

En la actualidad, estudios en animales y clínicos sobre el crecimiento guiado del fémur proximal medial comunican buenos resultados. ${ }^{12-17}$ No encontramos citas bibliográficas nacionales.

\section{Estudios experimentales}

McCarthy y cols. ${ }^{12}$ publican el primer estudio piloto en un modelo animal. Dividieron a 10 corderos en dos grupos de cinco: a un grupo, le colocaron un tornillo transfisario inferior en una de las caderas y, al otro, un tornillo en el cuello, sin cruzar la fisis, a modo de procedimiento simulado. En el grupo con el tornillo transfisario, el ACD disminuyó, en promedio, $11^{\circ}$. Esta diferencia fue significativa, representa una disminución del $8 \%$ aproximadamente del valor normal del ACD. d'Heurle y cols..$^{13}$ publicaron un estudio en animales cuyo objetivo era producir una deformidad en varo del fémur proximal. Realizaron una hemiepifisiodesis medial del fémur proximal con tornillo, con placa en 8 y con taladro percutáneo en cuatro corderos por grupo. La técnica con tornillo fue el único método que provocó una disminución significativa del ACD comparando con el lado contralateral (disminución promedio de $7^{\circ}$ ). Esta diferencia representa una disminución de alrededor del $6 \%$ del valor normal.

Ambas investigaciones ${ }^{12,13}$ nos dejan como interrogantes si los cambios son temporales y si se pueden revertir con la extracción del implante. Tienen las mismas limitaciones: una muestra pequeña y el uso de un modelo cuadrúpedo.

\section{Estudios clínicos}

Portinaro y cols. ${ }^{16}$ publicaron un protocolo de manejo de niños con parálisis cerebral, en el que dan lugar a la hemiepifisiodesis medial de cadera. Es una publicación solo informativa, no comunica resultados específicos.

Wei-Chun Lee y cols. ${ }^{14}$ reportan una serie de nueve niños (promedio de edad, 6 años), con parálisis cerebral GMFCS IV y V. Cinco fueron sometidos a cirugía de cadera unilateral y cuatro, a cirugía bilateral. A todos se les realizó una LPB y un crecimiento guiado con tornillo canulado transfisario medial de $7 \mathrm{~mm}$ de diámetro, en las caderas afectadas. El seguimiento promedio fue de 3.8 años. Los autores midieron el ACD antes de la cirugía y tres veces después de ella (una a los 3 meses y otras dos, al año y al segundo año). Los autores comunican una disminución promedio significativa de $12^{\circ}$ en el ACD, que representa un 7\%. Este resultado es equivalente al de los estudios experimentales ya mencionados. ${ }^{12,13}$ Asimismo, en sus tablas, informan un ACD promedio de $173,3^{\circ}$ a los 
tres meses de la operación, $166,4^{\circ}$ al año y $162,7^{\circ}$ a los dos años. La diferencia entre la medición a los tres meses y al año fue significativa $(\mathrm{p}<0,001$, potencia $=0,86)$, pero no entre la del año y los dos años $(\mathrm{p}=0,15$, potencia $=0,81)$. Después del primer año de la intervención, los cambios del ACD no mostraron una progresión conforme crecía el paciente. Esto se correlaciona con datos extraídos de las tablas del estudio respecto del IR durante el seguimiento: $7,7 \%$ buenos (IR <24); 61,5\% regulares (IR 25-39); $23 \%$ pobres (IR 40-59) y 7,7\% fracaso (IR >60). Tal vez estos resultados se debieron a la LPB más que a la hemiepifisiodesis.

A diferencia de nuestra serie, en su ensayo, Wei-Chun Lee y cols. ${ }^{14}$ no informan complicaciones, pero reportan una migración distal de los tornillos con el paso del tiempo, en todos los casos, tal como ocurrió en nuestro estudio.

Portinaro y cols. ${ }^{17}$ publican la serie más grande de hemiepifisiodesis medial en la cadera espástica, que incluyó a 28 pacientes (7 GMFCS III, 9 GMFCS IV, 12 GMFCS V) operados de ambas caderas, mediante la LPB más la colocación de un tornillo medial de 4,5 mm en el fémur proximal, con un seguimiento mínimo de cinco años. Evaluaron clínica y radiográficamente a los pacientes antes de la cirugía, y a los seis meses, uno y cinco años. En tres de los seis pacientes operados de ambas caderas, detectaron la migración distal de los tornillos a los 24 meses de la cirugía. En uno de ellos, con migración de ambos tornillos, uno de los tornillos se rompió ("descabezó") en el momento del recambio. El ACD disminuyó, en promedio, $14^{\circ}$ a los cinco años. Estos autores reportan una "disminución importante" del IR a los cinco años, pero el valor absoluto promedio es del 9\%. Asimismo, sugieren que la hemiepifisiodesis medial con tornillo puede ser útil para prevenir la luxación progresiva de la cadera, incluso en pacientes de alto riesgo. Sin embargo, en su serie, la displasia espástica progresó a luxación en cuatro de los 12 pacientes GMFCS V, que requirieron cirugía reconstructiva.

Torode y Young ${ }^{15}$ evalúan el crecimiento guiado en el fémur proximal con un tornillo transfisario medial para el tratamiento del caput valgum asociado a la displasia del desarrollo de la cadera. Excluyeron a pacientes con displasia de cadera secundaria a cuadros neuromusculares u otras enfermedades teratológicas. Evaluaron 13 caderas en 11 pacientes, con un promedio de edad de 9 años y 3 meses en el momento de la cirugía. El tiempo medio de seguimiento fue de cinco años. Consideraron mediciones radiográficas tanto femorales como acetabulares. El ACD disminuyó significativamente, de $173^{\circ}$ en promedio, antes de la cirugía, a $158^{\circ}$ en el seguimiento máximo. El ACD disminuyó un promedio del 8,6\%. Los autores concluyen en que la identificación precoz de un caput valgum se puede tratar con un tornillo transfisario medial de $7 \mathrm{~mm}$ de diámetro en el fémur proximal. La mejora en la orientación de la fisis y en el ACD refleja la eficacia de los principios de crecimiento guiado en el fémur proximal.

En un estudio sobre la prevención de cirugías óseas en niños con parálisis cerebral y displasia espástica de la cadera, Presedo y cols.$^{20}$ aportan evidencia científica consistente. Operaron a 65 pacientes ( 129 caderas), con una media de la edad de 4 años en el momento de la cirugía y un seguimiento promedio de 10 años. El $72 \%$ eran no deambuladores. Los resultados fueron los siguientes: $55 \%$ buenos; $14 \%$ regulares; $3 \%$ pobres y $27 \%$ fracasos. Una nueva LPB no se considera fracaso.

Nuestro estudio comunica los resultados de dos series de pacientes pediátricos similares, con caderas espásticas en riesgo. Compara los resultados entre la LPB más la colocación de un tornillo excéntrico transfisario medial en el fémur proximal, buscando una hemiepifisiodesis que provoque una deformidad en varo, como coadyuvante y solo la LPB. No hubo diferencias significativas entre las series a poco más de cuatro años de seguimiento promedio en cuanto a parámetros clínicos, como el test de Rang, entre ambas caderas, y radiográficos, como el IR de ambas caderas y el ACD de la cadera derecha. El ACD de la cadera izquierda presentó una diferencia estadísticamente significativa (p 0,02). Esto lo explica una complicación: un paciente de la serie A desarrolló un cierre fisario medial definitivo de la cadera izquierda. En esta serie, hubo dos complicaciones no menores, sobre un total de 20 caderas $(10 \%)$. Una ruptura del tornillo durante la colocación y un paciente con dolor persistente que requirió una nueva cirugía para retirar el implante con el posterior cierre fisario definitivo medial en la cadera izquierda.

Con respecto al propósito de este estudio, la colocación de un tornillo transfisario, excéntrico, medial de 4,5 mm en el fémur proximal de pacientes con PCI no fue eficaz para producir cambios morfológicos en las caderas. No logró aportar cambios al comparar los delta de medianas del IR y el ACD preoperatorios y posoperatorios en las series A vs. B (Tabla 3, Figura 8). Se hallaron solo diferencias estadísticamente significativas en el delta de mediana del ACD de la cadera izquierda (-5 vs. 0; p 0,02). La morfología del fémur proximal no se modificó, a excepción del paciente que sufrió el cierre fisario después de retirar el tornillo, en su cadera izquierda (Figura 7).

En cuanto a los objetivos generales, no se pudo confirmar que la colocación de un tornillo medial en la cadera sea un gesto coadyuvante útil a la LPB para mejorar la displasia de la cadera espástica en el corto plazo (promedio 51 meses). Se pudo determinar que la colocación de un tornillo medial en la cadera de pacientes con PCI no es un gesto inocuo, exento de complicaciones. Todos los tornillos migraron hacia distal durante el seguimiento. 
Tabla 3. Comparación del delta de medianas

\begin{tabular}{|c|c|c|c|c|}
\hline & $\begin{array}{c}\text { Total } \\
\mathrm{n}=18\end{array}$ & $\begin{array}{c}\text { Grupo A } \\
\mathbf{n}=\mathbf{1 0}\end{array}$ & $\begin{array}{c}\text { Grupo B } \\
\mathbf{n}=\mathbf{8}\end{array}$ & $\mathbf{p}$ \\
\hline Delta de mediana del test de Rang CD & 25 (RIC 15-35) & 23 (RIC 15-30) & 28 (RIC 18-35) & 0,3 \\
\hline Delta de mediana del test de Rang CI & 25 (RIC 20-30) & 25 (RIC 25-25) & 20 (RIC 15-30) & 0,3 \\
\hline Delta de mediana del IR CD & -1 (RIC de -10 a -1$)$ & $3(\mathrm{RIC}$ de -12 a 21$)$ & -2 (RIC de -9 a 8) & 0,56 \\
\hline Delta de mediana del IR CI & -5 (RIC de -13 a 38$)$ & -9 (RIC de -23 a 5$)$ & -5 (RIC de -6 a 38$)$ & 0,3 \\
\hline Delta de mediana del ACD CD & 0 (RIC de -5 a 5$)$ & $-1(\mathrm{RIC}$ de -7 a 5$)$ & 0 (RIC 0-0) & 0,2 \\
\hline Delta de mediana del ACD CI & $-2(\mathrm{RIC}$ de -5 a 3$)$ & -5 (RIC de -10 a 0$)$ & 0 (RIC de -2 a 3) & 0,02 \\
\hline
\end{tabular}

$\mathrm{CD}=$ cadera derecha, $\mathrm{CI}$ = cadera izquierda, $\mathrm{IR}=$ índice de Reimer, $\mathrm{ACD}=$ ángulo cervicodiafisario.

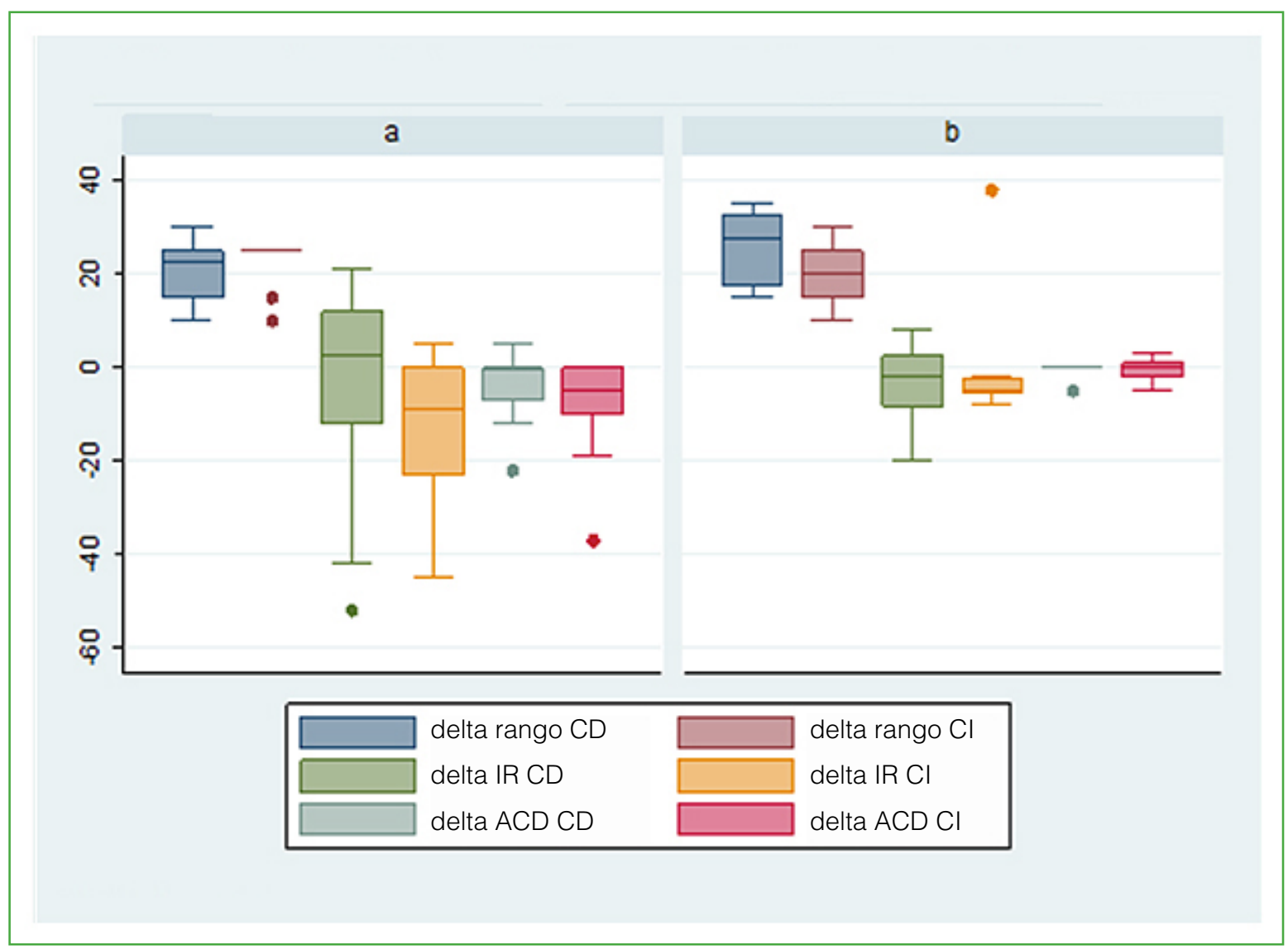

Figura 8. Delta de medianas de las medidas preoperatorias y posoperatorias, por grupos. ACD = ángulo cervicodiafisario del fémur proximal; $\mathrm{CD}$ = cadera derecha, $\mathrm{CI}=$ cadera izquierda, $\mathrm{IR}=$ índice de Reimer.

Como las morbilidades periquirúrgicas en esta población son elevadas y, dados los costos adicionales, se decidió no cambiar tornillos ni agregar otro tornillo durante el seguimiento cuando se detectó el desprendimiento fisario del tornillo. Esto indicaría que la epifisiodesis no se produjo; por lo tanto, es esperable no observar modificaciones en las mediciones radiográficas. Algunos autores consideran que el desplazamiento del tornillo luego de uno o dos años no afecta el resultado final. ${ }^{14}$ 
Nuestro estudio tiene algunas limitaciones, como el reducido número de pacientes y el seguimiento acotado, ya que ningún paciente llega a desarrollar el pico de crecimiento prepuberal, momento de mayor deterioro, recidiva y progresión de la subluxación. Pero, en cuatro años de seguimiento promedio, no se observaron diferencias significativas en los porcentajes de pacientes con diferentes IR: serie A, 55\% buenos, $25 \%$ regulares, $15 \%$ pobres y $5 \%$ fracaso; serie $\mathrm{B}, 50 \%$ buenos, $31 \%$ regulares, $13 \%$ pobres y $6 \%$ fracaso (p 0,1 , diferencia no significativa) ${ }^{20}$

Si consideramos la bibliografía revisada, ${ }^{12-17}$ el concepto de crecimiento guiado en el fémur proximal se vuelve muy atractivo, ya que promete una potencial solución biológica y menos cruenta a los problemas de la displasia espástica de cadera en pacientes con PCI. Sin embargo, analizando exhaustivamente las diferentes investigaciones y el contraste con nuestros resultados, nos surgen dudas. Todos los estudios ${ }^{12-17}$ coinciden en mostrar una disminución del ACD del 6-8\% del valor inicial. En caderas que no están sujetas a las fuerzas deformantes de los músculos espásticos, tal vez, una modificación de alrededor del 8\% del ACD sea suficiente para cambiar la evolución natural de un caput valgum como secuela de una displasia del desarrollo de la cadera, aunque parece poco probable que lo sea para caderas espásticas. Las caderas espásticas son el resultado del desequilibrio muscular. La mayor anteversión y el valgo del cuello femoral provocan un aumento progresivo de la displasia acetabular. Todo se agrava aún más por la falta de carga. ${ }^{21} \mathrm{Si}$ la fisiopatología de la cadera espástica es extrafisaria: ¿por qué estaría en la fisis la respuesta? Con respecto a las inferencias de los estudios, ${ }^{14,17}$ ¿cómo se puede asegurar que las mejoras del 6-8\% observadas en el ACD, particularmente durante el primer año posterior a la cirugía, se deben a la hemiepifisiodesis medial del fémur proximal y no al efecto que provoca la LPB? Además, existen desafíos asociados con la precisión y la reproducibilidad de las mediciones radiográficas en niños, ya que puede ser difícil lograr una posición óptima para tomarles las radiografías.

\section{CONCLUSIONES}

La colocación de un tornillo excéntrico medial transfisario de 4,5 mm en el fémur proximal de pacientes con PCI no fue eficaz para producir cambios morfológicos. Para el tratamiento preventivo de la cadera espástica, no recomendamos agregar la colocación de un tornillo transfisario medial en el fémur proximal, ya que no es un método inocuo, aumenta el riesgo de complicaciones y la evidencia científica aún no es contundente.

Dados su potencial beneficio y su relativa baja morbilidad, el crecimiento guiado del fémur proximal debe ser objeto de nuevas y mayores investigaciones, con más casuística, casos y controles y seguimiento prolongado.

Conflicto de intereses: Los autores no declaran conflictos de intereses.

ORCID de F. Lucioni: https://orcid.org/0000-0002-9097-5113

ORCID de J. P. Cucchiara: https://orcid.org/0000-0002-3105-7478

\section{BIBLIOGRAFÍA}

1. Access Economics Pty Ltd. The economic impact of cerebral palsy in Australia in 2007. Report. Australia: Cerebral Palsy Australia 2008. https://cpaustralia.com.au/media/20379/access_economics_report.pdf

2. Mandaleson A, Lee Y, Kerr C, Graham HK. Classifying cerebral palsy: are we nearly there? J Pediatr Orthop 2015;35(2):162-6. https://doi.org/10.1097/BPO.0000000000000222

3. Cooke PH, Cole WG, Carey RP. Dislocation of the hip in cerebral palsy. Natural history and predictability. $J$ Bone Joint Surg Br 1989;71(3):441-6. PMID: 2722938

4. Miller F, Cardoso Dias R, Dabney KW, Lipton GE, Triana M. Soft-tissue release for spastic hip subluxation in cerebral palsy. J Pediatr Orthop 1997;17(5):571-84. https://doi.org/10.1097/00004694-199709000-00003 
5. Flynn JM, Millar F. Management in hip disorders in patients with cerebral palsy. J Am Acad Orthop Surg 2002;10(3):198-209. https://doi.org/10.5435/00124635-200205000-00006

6. Andrisano A, Marchiodi L, Preitano M. Epiphyseodesis of the great trochanter. Ital J Orthop Traumatol 1986;12(2):217-22. PMID: 3793460

7. Davids JR, Valadie AL, Ferguson RL, Bray EW 3rd, Allen BL Jr. Surgical management of ankle valgus in children: use of a transphyseal medial malleolar screw. J Pediatr Orthop 1997;17(1):3-8. PMID: 8989691

8. Stevens PM, Belle RM. Screw epiphysiodesis for ankle valgus. J Pediatr Orthop 1997;17(1):9-12. PMID: 8989692

9. Métaizeau JP, Wong-Chung J, Bertrand H, Pasquier P. Percutaneous epiphysiodesis using transphyseal screws (PETS). J Pediatr Orthop 1998;18(3):363-9. PMID: 9600565

10. Beals RK, Shea M. Correlation of chronological age and bone age with the correction of ankle valgus by surface epiphysiodesis of the distal medial tibial physis. J Pediatr Orthop B 2005;14(6):436-8. https://doi.org/10.1097/01202412-200511000-00009

11. Stevens PM, Novais EN. Multilevel guided growth for hip and knee varus secondary to chondrodysplasia. J Pediatr Orthop 2012;32(6):626-30. https://doi.org/10.1097/BPO.0b013e3182567a79

12. McCarthy JJ, Noonan KJ, Nemke B, Markel M. Guided growth of the proximal femur: a pilot study in the lamb model. J Pediatr Orthop 2010;30(7):690-4. https://doi.org/10.1097/BPO.0b013e3181edef71

13. d'Heurle A, McCarthy J, Klimaski D, Stringer K. Proximal femoral growth modification: effect of screw, plate, and drill on asymmetric growth of the hip. J Pediatr Orthop 2018;38(2):100-4. https://doi.org/10.1097/BPO.0000000000000771

14. Wei-Chun Lee, Hsuan-Kai Kao, Wen-E Yang, Pei-Chi Ho, Chia-Hsieh Chang. Guided growth of the proximal femur for hip displacement in children with cerebral palsy. J Pediatr Orthop 2016;36(5):511-5. https://doi.org/10.1097/BPO.0000000000000480

15. Torode IP, Young JL. Caput valgum associated with developmental dysplasia of the hip: management by transphyseal screw fixation. J Child Orthop 2015;9(5):371-9. https://doi.org/10.1007/s11832-015-0681-9

16. Portinaro N, Panou A, Gagliano N, Pelillo F. D.D.S.H.: Developmental dysplasia of the spactic hip: Strategies of management in cerebral palsy. A new suggestive algorithm. Hip Int 2009;19(Suppl 6):S69-74. https://doi.org/10.1177/112070000901906s12

17. Portinaro N, Turati M, Cometto M, Bigoni M, Davids JR, Panou A. Guided growth of the proximal femur for the management of hip dysplasia in children with cerebral palsy. J Pediatr Orthop 2019;39(8):e622-8. https://doi.org/10.1097/BPO.0000000000001069

18. Carreño-Mora F, Ortiz-Corredor F, Espinosa-García E, Pérez-Hernández CE. Validación de un instrumento para evaluar la carga del cuidador en parálisis cerebral. Rev Salud Pública (Bogota) 2015;17(4):578-88. https://doi.org/10.15446/rsap.v17n4.35593

19. Dindo D, Demartines N, Clavien PA. Classification of surgical complications. A new proposal with evaluation in a cohort of 6336 patients and results of a survey. Ann Surg 2004;240(2):205-13. https://doi.org/10.1097/01.sla.0000133083.54934.ae

20. Presedo A, Oh CW, Dabney KW, Miller F. Soft-tissue releases to treat spastic hip subluxation in children with cerebral palsy. J Bone Joint Surg Am 2005;87(4):832-41. https://doi.org/10.2106/JBJS.C.01099

21. García Mata S, Duart Clemente J. Cirugía preventiva de la luxación de cadera espástica. En: Martínez Caballero I, Abad Lara JA (eds.). Parálisis cerebral infantil. Manejo de las alteraciones músculo-esqueléticas asociadas. Madrid: Ergon; 2016:79-92. 